portant factor in the calculations. There is agreement between the present mean life results and the recent beam-foil measurements of Heroux [3] and Bromander [5] and with the electron impact phase-shift method results of Lawrence [4].

\section{References}

1. E. L. Chupp. L.W.Dotchin and D.J.Pegg, Phys. Rev. 175 (1968) 44 .
2. P.C.Rogers, FRANTIC, M.I.T. Lab. for Nucl. Sci., Report 76 (1962).

3. L. Heroux, Phys. Rev. 180 (1969) 1.

4. G. M. Lawrence and B.D. Savage, Phys. Rev. 141 (1966) 67 .

5. J. Bromander, R. Buchta and L. Lundin, Phys. Letters 29A (1969) 523 .

6. P.Westhaus and O.Sinanoğlu, Phys. Rev. 183 (1969) 56.

7. A.W.Weiss, private communication.

8. A.W.Weiss, Phys. Rev. 162 (1967) 71.

\title{
INJECTION AND MOBILITY OF POTASSIUM IONS IN LIQUID HELIUM *
}

\author{
G. G. IHAS and T. M. SANDERS Jr. \\ H.M.Randall Physics Labs, Universily of Michigan, \\ Ann Arbor, Michigan, USA
}

Received 25 March 1970

\begin{abstract}
Potassium ions from a hot tungsten wire are injected into He II; their mobility is found to be two to three percent lower than that of 'normal' positive ions.
\end{abstract}

Charged carriers produced in liquid helium along the track of an ionizing particle have been studied in a variety of experiments [1]. According to the model of Atkins [2], the positive carrier consists of a helium ion surrounded by a 'snowball' of solid helium of radius about $6 \AA$ and mass about $40 M_{\mathrm{He}}(160 \mathrm{amu})$. The solidification is caused by the electrostrictive pressure due to the Coulomb field of the central ion. Further, theory predicts that the mobility $(\mu)$ of such a carrier will be independent of its mass provided its recoil in a collision with an elementary excitation is negligible. We have tested this prediction by measuring the difference between the mobility of 'normal' positive carriers and positive carriers in which the central helium ion of the 'normal' complex is replaced by an ion of potassium. Since the Coulomb field is then the same as in the normal case and both masses are large compared to the roton mass, the Atkins model leads to equal mobilities.

Tungsten filament wire (General Electric type 218) doped with approximately $100 \mathrm{ppm}$ potassium becomes a source of potassium ions

\footnotetext{
* This work is supported in part by the US Atomic Energy Commission.
}

when heated. Vacuum emission mass spectrometry of these wires showed that after an initial cleanup period only electrons and $\mathrm{K}^{+}$ions were emitted. Preliminary operation of wires of $7 \mu \mathrm{m}$ diameter without any other sources present under He II [3,4], established that positive ions could be injected into the liquid, even when the source voltage was well below threshold for ionization of helium, with a mobilitiy close to that of the 'normal' ion [1]. There seems to be no mechanism for the production of any ion other than potassium. Therefore, an apparatus to directly measure the difference of the mobilities of the two ions was constructed.

Two filament wires $S_{2}$ and a 210 Po radioactive source $S_{1}$ were mounted at the source (top) end of a time-of -flight spectrometer, in a cell in which the helium level could be maintained for optimum wire operation. The mobility apparatus (see insert in fig. 1) consisted of sources $\left(S_{1}\right.$ and $\left.S_{2}\right)$, two-grid gate $\left(G_{1}\right.$ and $\left.G_{2}\right)$, $2.5 \mathrm{~cm}$ drift region with field homogenizers $\left(\mathrm{H}_{1}, \mathrm{H}_{2}\right.$ and $\left.\mathrm{H}_{3}\right)$, Frisch grid $\left(\mathrm{G}_{\mathrm{F}}\right)$, and collector (C) with guard ring (R); current pulses were detected using a fast FET electrometer, timeaveraging computer, and $X Y$ plotter. Carriers of either polarity, coming from either radioac- 


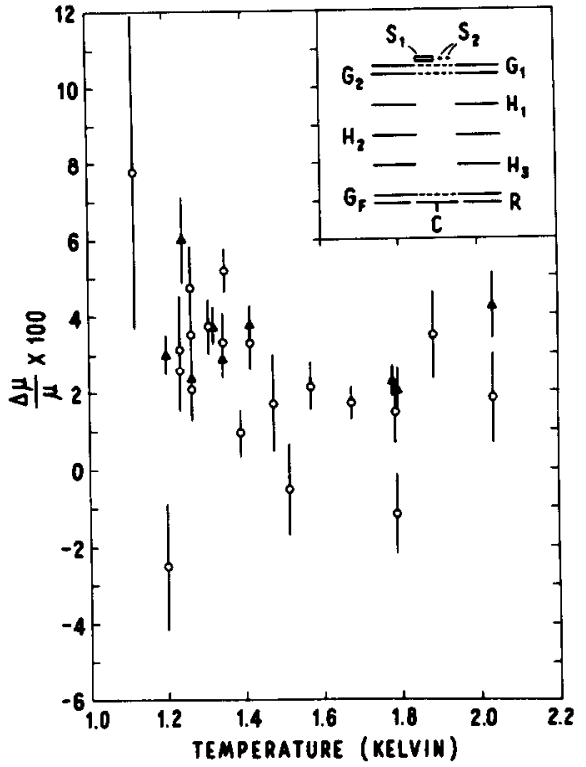

Fig. 1. Fractional difference in mobility between potassium ions and 'normals ions. The circles and triangles denote data taken using the first and second methods (see text), respectively. The insert shows the time-of-flight cell.

tive or hot-wire source, could be studied individually by appropriately setting the various electrode potentials. When comparisons of times-of-flight from the two different sources were being made, care was taken that the power dissipated in the hot-wire remained constant, to insure that convective effects were identical during both measurements. Also, all electric fields were kept the same except those between $\mathrm{S}_{1}, \mathrm{~S}_{2}$, and $\mathrm{G}_{1}$ (one source being reverse biased while the other was forward biased). Data were taken in two different ways: in the first, one signal at a time was summed in the computer, a time-of-flight was obtained for each species, and the results were compared later. In the second (differ ential) method, alternate signals from the two sources were electronically subtracted in the memory, directly yielding the dif ference of the two mobilities.

The results for positive ions from the two types of sources are shown in fig. 1. When a similar comparison is made of the times-of flight of negative carriers no difference is detected, even though the negative carrier is more sensitive to effects such as convection than positives. Within the precision of our data, the mobility of the potassium ions appears to be lower by 2 or $3 \%$ throughout the temperature range studied $\left(1.1-2.1^{\circ} \mathrm{K}\right)$. The error bars represent the total error for each fractional dif ference measurement.

This result, that a change in the mass of the core ion from 4 (or 8) amu to about 40 amu produces only a small change in mobility, is generally consistent with the electrostrictive model of the ion and present theory of mobility [5]. A detailed comparison must, however, await a theory of residual recoil corrections in the roton-scattering region.

We are grateful to J. G. King for suggesting to us that a hot wire might be used as a source of alkali ions. We also wish to acknowledge the as sistance of S. R. Ryan, whose equipment was used in these measurements.

\section{References}

1. For a survey of such experiments see R. J. Donnelly, Experimental superfluidity (University of Chicago Press, 1967).

2. K. R. Atkins, Phys. Rev. 116 (1959) 1339.

3. G. E. Spangler and F. L. Hereford, Phys. Rev. Letters 20 (1968) 1229.

4. G. P. Lemieux and A. C. Leonard, Mechanical Engineering 3 (1968) 74 .

5. G. Baym, R. G. Barrera and C. J. Pethick, Phys. Rev. Letters 22 (1969) 20. 